\title{
Asymptotic behavior and threshold of a stochastic SIQS epidemic model with vertical transmission and Beddington-DeAngelis incidence
}

\author{
Yang Chen ${ }^{1}$ and Wencai Zhao ${ }^{1 *}$ (D)
}

"Correspondence:

zhaowencai@sdust.edu.cn ${ }^{1}$ College of Mathematics and Systems Science, Shandong University of Science and Technology, Qianwangang Road 579, 266590 Qingdao, P.R. China

\section{Springer}

\begin{abstract}
This paper investigates a deterministic and stochastic SIQS epidemic model with vertical transmission and Beddington-DeAngelis incidence. Firstly, for the corresponding deterministic system, the global asymptotic stability of disease-free equilibrium and the endemic equilibrium is proved through the stability theory. Secondly, for the stochastic system, the threshold conditions which decide the extinction or permanence of the disease are derived. By constructing suitable Lyapunov functions, we investigate the oscillation behavior of the stochastic system solution near the endemic equilibrium. The results of this paper show that there exists a great difference between the deterministic and stochastic systems, which implies that the large stochastic noise contributes to inhibiting the spread of disease. Finally, in order to validate the theoretical results, a series of numerical simulations are presented.
\end{abstract}

MSC: $60 \mathrm{H} 10 ; 65 \mathrm{C} 30 ; 91 \mathrm{~B} 70$

Keywords: Beddington-DeAngelis incidence; Vertical transmission; Threshold; Asymptotic behavior

\section{Introduction}

Infectious diseases are the first killer of human beings. According to World Health Statistics Report 2016 published by the World Health Organization (WHO), there were 216 million cases of malaria in 2016, with more than 43,800 deaths. During the same period, 1.2 million people died of HIV-related diseases [1]. On 29 July 2019, the WHO Regional Office for Africa issued a report that the Democratic Republic of Congo has reported 1798 deaths of Ebola haemorrhagic fever from 2687 cases between August 2018 and July 2019. For this matter, several measures have been taken to prevent the spread of deadly infectious diseases all over the world such as malaria, cholera, and plague, which led countries to strengthen quarantine measures at their entry and exit ports, which were first taken by Venice authorities in the fourteenth century. The personnel of foreign ships arriving at their ports should be allowed to stay on board for 40 days. They are allowed to leave

(c) The Author(s) 2020. This article is licensed under a Creative Commons Attribution 4.0 International License, which permits use, sharing, adaptation, distribution and reproduction in any medium or format, as long as you give appropriate credit to the original author(s) and the source, provide a link to the Creative Commons licence, and indicate if changes were made. The images or other third party material in this article are included in the article's Creative Commons licence, unless indicated otherwise in a credit line to the material. If material is not included in the article's Creative Commons licence and your intended use is not permitted by statutory regulation or exceeds the permitted use, you will need to obtain permission directly from the copyright holder. To view a copy of this licence, visit http://creativecommons.org/licenses/by/4.0/ 
the ship for landing only after they have been inspected by the port authorities and no disease has been found. The reason is that if you suffer from an infectious disease, it can usually manifest within 40 days [2]. The original quarantine measure played a great role in preventing the spread of infectious diseases such as plague at that time. For infectious diseases to occur and spread in a certain population, they must have three basic elements: the source of infection, the route of transmission, and the susceptible population. Quarantine of infectious sources and timely treatment of infected persons are important measures to control the spread of diseases. Quarantine can greatly reduce the probability of contact between infective and susceptible individuals. In recent years, a large number of epidemic models have been used in the study of disease prevention and control strategies [3-14]. The influence of quarantine measures on the second epidemic of plague in Europe was studied in literature [15]. Similarly, strict inspection and quarantine are also effective measures to deal with animal and plant infectious diseases [16, 17]. In 2002, Hethcote et al. proposed a model with quarantine [18]:

$$
\left\{\begin{array}{l}
\frac{d S(t)}{d t}=A-u S(t)-\beta I(t) S(t)+\gamma I(t)+\varepsilon Q(t) \\
\frac{d I(t)}{d t}=\beta I(t) S(t)-\left(u+\alpha_{2}+\delta+\gamma\right) I(t) \\
\frac{d Q(t)}{d t}=\delta I(t)-\left(u+\alpha_{3}+\varepsilon\right) Q(t)
\end{array}\right.
$$

where $S(t), I(t), Q(t)$ represent the susceptible individuals, the infective but not isolated infected individuals, and the quarantined individuals, respectively. $A$ is the recruitment rate. $u$ denotes the natural mortality rate. $\gamma$ and $\varepsilon$ are the rates at which individuals recover and return to $S$ from $I$ and $Q$, respectively. $\delta$ is the rate constant for individuals leaving the infective individuals for the quarantined ones. $\alpha_{2}$ stands for the disease-related mortality rate of $I . \alpha_{3}$ represents the disease-related mortality rate of $Q$.

In the process of infectious disease modeling, infectious rate is an important indicator to describe the speed of disease transmission. According to the characteristics of different diseases transmission, choosing the appropriate infection rate is the basis of accurate modeling. The above bilinear incidence $\beta S(t) I(t)$ is used in the model in [18]. Wei et al. [19] proposed an SIQS model with saturation incidence $\frac{\beta S(t) I(t)}{1+\alpha I(t)}$. In reference [20], an SEIR model with Beddington-DeAngelis incidence $\frac{\beta S(t) I(t)}{1+m S(t)+n I(t)}$ and vertical transmission is considered. Obviously, if $m=0$, the incidence becomes to saturated incidence $\frac{\beta S(t) I(t)}{1+\alpha I(t)}$. Furthermore, when $m=n=0$, the Beddington-DeAngelis incidence rate degenerates into bilinear incidence rate $\beta S(t) I(t)$. In real life, the route of disease transmission is complex and diverse. During pregnancy or childbirth, the disease can be transmitted directly from the mother to the embryo or infant, i.e., vertical transmission [21]. Motivated by the above-mentioned work, this paper establishes the following epidemic model with vertical transmission and Beddington-DeAngelis incidence:

$$
\left\{\begin{aligned}
\frac{d S(t)}{d t}= & A-u S(t)-\frac{\beta I(t) S(t)}{1+m S(t)+n I(t)}+b(S(t)+Q(t))+(1-q) b I(t) \\
& +\gamma I(t)+\varepsilon Q(t), \\
\frac{d I(t)}{d t}= & \frac{\beta I(t) S(t)}{1+m S(t)+n I(t)}+q b I(t)-\left(u+\alpha_{2}+\delta+\gamma\right) I(t), \\
\frac{d Q(t)}{d t}= & \delta I(t)-\left(u+\alpha_{3}+\varepsilon\right) Q(t),
\end{aligned}\right.
$$


where $m$ and $n$ denote the parameters for measuring suppression effect, respectively. $b$ denotes the birth rate and $u>b . q$ represents the vertical transmission coefficient and $0<q<1$.

It is well known that environmental noise in nature always affects biological systems to a greater or lesser extent [22-24], so the limitation of deterministic model is inevitable. In view of the fact that stochastic systems can better describe practical problems, a large number of stochastic differential equation models have been applied to study various ecosystems in recent years. For instance, the influence of white noise on the contact coefficient is considered in [25-32], and the Markov chain is used in [33-37] to represent the interference of colored noise on the system. In order to describe the dramatic changes in natural environment such as earthquakes and floods, scholars explain these phenomena by adding Lévy jump coupling to deterministic models [38-41].

In this paper, we assume that the contact rate is disturbed by white noise. That is $\beta \rightarrow \beta+$ $d B(t)$, then model (2) is transformed into the following stochastic SIQS epidemic model:

$$
\left\{\begin{aligned}
d S(t)= & \left(A-u S(t)-\frac{\beta I(t) S(t)}{1+m S(t)+n I(t)}+b(S(t)+Q(t))+(1-q) b I(t)\right. \\
& +\gamma I(t)+\varepsilon Q(t)) d t-\frac{\sigma I(t) S(t)}{1+m S(t)+n I(t)} d B(t) \\
d I(t)= & \left(\frac{\beta I(t) S(t)}{1+m S(t)+n I(t)}+q b I(t)-\left(u+\alpha_{2}+\delta+\gamma\right) I(t)\right) d t \\
& +\frac{\sigma I(t) S(t)}{1+m S(t)+n I(t)} d B(t) \\
d Q(t)= & \left(\delta I(t)-\left(u+\alpha_{3}+\varepsilon\right) Q(t)\right) d t
\end{aligned}\right.
$$

where $\sigma^{2}$ is the intensity of the white noise, $B(t)$ is the standard Brownian motion defined on a complete probability space $\left(\Omega, \mathcal{F},\{\mathcal{F}\}_{t \geq 0}, \mathcal{P}\right)$.

This paper is organized as follows. We give some auxiliary results and related lemmas in Sect. 2. We study the existence and asymptotic stability of equilibria in a deterministic system in Sect. 3. And in Sect. 4, the threshold condition for disease extinction and permanence of a stochastic system is given. In Sect. 5, the asymptotic behavior of the stochastic system is discussed. In Sect. 6, the correctness of our conclusions are verified by numerical simulations.

\section{Preliminaries}

Throughout this paper, we let $R_{+}^{3}=\left\{x_{i}>0, i=1,2,3\right\}$. For any integrable function $f$ on $[0,+\infty)$, we define $\langle f(t)\rangle=\frac{1}{t} \int_{0}^{t} f(\pi) \mathrm{d} \pi$. From system (3), we can get

$$
\frac{d(S(t)+I(t)+Q(t))}{d t}=A-(u-b)(S(t)+I(t)+Q(t))-\alpha_{2} I(t)-\alpha_{3} Q(t) .
$$

This shows that

$$
S(t)+I(t)+Q(t) \leq \frac{A}{u-b}+\left(S(0)+I(0)+Q(0)-\frac{A}{u-b}\right) e^{-(u-b) t} .
$$

Now, we define

$$
\Gamma=\left\{(S(t), I(t), Q(t)) \in R_{+}^{3}: S(t)+I(t)+Q(t) \leq \frac{A}{u-b}\right\} .
$$

Obviously, the region $\Gamma$ is a positively invariant set. 
Lemma 2.1 For any initial value $(S(0), I(0), Q(0)) \in R_{+}^{3}$, system (3) has a unique solution $(S(t), I(t), Q(t))$ on $t \geq 0$, and the solution will remain in $R_{+}^{3}$ with probability one.

Proof The coefficients of system (3) satisfy the local Lipschitz condition, for any given initial value $(S(0), I(0), Q(0)) \in R_{+}^{3}$, there is a unique local solution $(S(t), I(t), Q(t))$ on interval $\left[0, \tau_{e}\right)$, where $\tau_{e}$ denotes the explosion time [42]. If we want to prove the solution is global, we just need to prove $\tau_{e}=\infty$ a.s. We assume that $k_{0}>0$ such that $S(0)>\frac{1}{k_{0}}, I(0)>\frac{1}{k_{0}}$, $Q(0)>\frac{1}{k_{0}}$. Let $k>k_{0}$, the stopping time is defined as

$$
\tau_{k}=\inf \left\{t \in\left[0, \tau_{e}\right): S(t) \leq \frac{1}{k} \text { or } I(t) \leq \frac{1}{k} \text { or } Q(t) \leq \frac{1}{k}\right\}
$$

and we know $\tau_{k}$ is increasing as $k \rightarrow \infty$. Let $\tau_{\infty}=\lim _{k \rightarrow+\infty} \tau_{k}$, thus $\tau_{\infty} \leq \tau_{e}$. Therefore, we only need to certify that $\tau_{\infty}=\infty$. Otherwise, there is a positive constant $T>0$ and $\varepsilon \in(0,1)$ such that $P\left\{\tau_{\infty}<\infty\right\}>\varepsilon$. Hence, there exists an integer $k_{1}>k_{0}$ satisfying $P\left\{\tau_{k} \leq T\right\} \geq \varepsilon$ for any positive $k>k_{1}$.

Define a $C^{2}$-function $V_{1}$ :

$$
V_{1}(S(t), I(t), Q(t))=-\ln \frac{S(t)}{C_{1}}-\ln \frac{I(t)}{C_{1}}-\ln \frac{Q(t)}{C_{1}},
$$

where $C_{1}=\max \left\{S(0)+I(0)+Q(0), \frac{A}{u-b}\right\}$. Applying Itô’s formula on $V_{1}$, we obtain

$$
d V_{1}=L V_{1} d t+\frac{\sigma(I(t)-S(t))}{1+m S(t)+n I(t)} d B(t)
$$

where

$$
\begin{aligned}
L V_{1}= & -\frac{A+b Q(t)+(1-q) b I(t)+\gamma I(t)+\varepsilon Q(t)}{S(t)}+u-b+\frac{\beta I(t)}{1+m S(t)+n I(t)} \\
& -\frac{\beta S(t)}{1+m S(t)+n I(t)}+\left(u+\alpha_{2}+\delta+\gamma-q b\right)-\frac{\delta I(t)}{Q(t)}+\left(u+\alpha_{3}+\varepsilon\right) \\
& +\frac{\sigma^{2}\left(I^{2}(t)+S^{2}(t)\right)}{2(1+m S(t)+n I(t))^{2}} \\
\leq & 3 u+\alpha_{2}+\delta+\gamma+\alpha_{3}+\varepsilon+\frac{\beta I(t)}{1+m S(t)+n I(t)}+\frac{\sigma^{2}\left(I^{2}(t)+S^{2}(t)\right)}{2} \\
\leq & 3 u+\alpha_{2}+\delta+\gamma+\alpha_{3}+\varepsilon+\beta C_{1}+\sigma^{2} C_{1}^{2}:=C_{2} .
\end{aligned}
$$

The following proof process is similar to [43] and we omitted it. The proof is completed.

\section{The stability analysis of deterministic system (2)}

For system (2), whether the disease can be eradicated is widely concerned. Therefore, it is of great significance to discuss the existence and stability of the equilibrium. First, according to the method of literature [44], we define

$$
R_{0}=\frac{A \beta}{(A m+u-b)\left(u+\alpha_{2}+\delta+\gamma-q b\right)} .
$$


Let $\frac{d S(t)}{d t}=0, \frac{d I(t)}{d t}=0, \frac{d Q(t)}{d t}=0$. We obtain two equilibria for system (2): $E_{0}=\left(\frac{A}{u-b}, 0,0\right)$, $E^{*}=\left(S^{*}, I^{*}, Q^{*}\right)$, where

$$
\begin{aligned}
& I^{*}=\frac{A-\frac{(u-b)\left(u+\alpha_{2}+\delta+\gamma-q b\right)}{\beta-m\left(u+\alpha_{2}+\delta+\gamma-q b\right)}}{n \frac{(u-b)\left(u+\alpha_{2}+\delta+\gamma-q b\right)}{\beta-m\left(u+\alpha_{2}+\delta+\gamma-q b\right)}+\left(u-b+\alpha_{2}\right)+\frac{u-b+\alpha_{3}}{u+\alpha_{3}+\varepsilon} \delta}, \\
& S^{*}=\frac{\left(u+\alpha_{2}+\delta+\gamma-q b\right)\left(1+n I^{*}\right)}{\beta-m\left(u+\alpha_{2}+\delta+\gamma-q b\right)}, \\
& Q^{*}=\frac{\delta}{u+\alpha_{3}+\varepsilon} I^{*} .
\end{aligned}
$$

When $R_{0}>1, E^{*}=\left(S^{*}, I^{*}, Q^{*}\right)$ is a unique endemic equilibrium. If $E_{0}=\left(\frac{A}{u-b}, 0,0\right)$ is globally asymptotically stable, the disease will become extinct. If $E^{*}=\left(S^{*}, I^{*}, Q^{*}\right)$ is globally asymptotically stable, the disease will be permanent. Next, we use the eigenvalue method to study the stability of $E_{0}$ and $E^{*}$, respectively, so as to obtain the threshold condition of whether the disease is extinct or not.

Theorem 3.1 For system (2), we come to a conclusion:

(i) If $R_{0}<1$, then it has a disease-free equilibrium $E_{0}=\left(\frac{A}{u-b}, 0,0\right)$, which is globally asymptotically stable.

(ii) If $R_{0}>1$, then it has an endemic equilibrium $E^{*}=\left(S^{*}, I^{*}, Q^{*}\right)$, which is globally asymptotically stable.

Proof (i) The Jacobian matrix of $E_{0}=\left(\frac{A}{u-b}, 0,0\right)$ is

$$
J_{0}=\left(\begin{array}{ccc}
b-u & -\frac{A \beta}{A m+u-b}+(1-q) b+\gamma & b+\varepsilon \\
0 & \frac{A \beta}{A m+u-b}+q b-\left(u+\alpha_{2}+\delta+\gamma\right) & 0 \\
0 & \delta & -\left(u+\alpha_{3}+\varepsilon\right)
\end{array}\right) \text {. }
$$

Hence, the characteristic equation can be obtained as follows:

$$
(\lambda-b+u)\left[\lambda-\frac{A \beta}{A m+u-b}-q b+\left(u+\alpha_{2}+\delta+\gamma\right)\right]\left[\lambda+\left(u+\alpha_{3}+\varepsilon\right)\right]=0 .
$$

The three eigenvalues are $\lambda_{1}=b-u<0, \lambda_{2}=\frac{A \beta}{A m+u-b}-\left(u+\alpha_{2}+\delta+\gamma-q b\right)$, and $\lambda_{3}=$ $-\left(u+\alpha_{3}+\varepsilon\right)<0$. Therefore, $E_{0}=\left(\frac{A}{u-b}, 0,0\right)$ is locally asymptotically stable if $R_{0}<1$.

Let $V_{2}(S(t), I(t), Q(t))=I(t)$ as a Lyapunov function, we can calculate

$$
\begin{aligned}
\frac{d V_{2}}{d t} & =\frac{\beta I(t) S(t)}{1+m S(t)+n I(t)}+q b I(t)-\left(u+\alpha_{2}+\delta+\gamma\right) I(t) \\
& \leq\left[\frac{A \beta}{A m+u-b}-\left(u+\alpha_{2}+\delta+\gamma-q b\right)\right] I(t) \\
& =\left(u+\alpha_{2}+\delta+\gamma-q b\right)\left(R_{0}-1\right) I(t) .
\end{aligned}
$$

Hence, $\frac{d V_{2}}{d t} \leq 0$ when $R_{0}<1$, and it follows that $V_{2}(S(t), I(t), Q(t))$ is bounded and nonincreasing. By LaSalle's invariance principle, the disease-free equilibrium $E_{0}=\left(\frac{A}{u-b}, 0,0\right)$ is globally asymptotically stable. 
(ii) The Jacobian matrix of $E^{*}=\left(S^{*}, I^{*}, Q^{*}\right)$ is

$$
J^{*}=\left(\begin{array}{ccc}
a_{11}{ }^{*} & a_{12}{ }^{*} & b+\varepsilon \\
a_{21}{ }^{*} & a_{22}{ }^{*} & 0 \\
0 & \delta & a_{33}{ }^{*}
\end{array}\right),
$$

where

$$
\begin{aligned}
& a_{11}^{*}=\frac{-\beta I^{*}\left(1+n I^{*}\right)}{\left(1+m S^{*}+n I^{*}\right)^{2}}+b-u=-a_{21}{ }^{*}+b-u<0, \\
& a_{12}{ }^{*}=\frac{-\beta S^{*}\left(1+m S^{*}\right)}{\left(1+m S^{*}+n I^{*}\right)^{2}}+(1-q) b+\gamma=-a_{22}{ }^{*}-\left(u+\alpha_{2}+\delta-b\right)<0, \\
& a_{21}{ }^{*}=\frac{\beta I^{*}\left(1+n I^{*}\right)}{\left(1+m S^{*}+n I^{*}\right)^{2}}>0, \\
& a_{22}{ }^{*}=\frac{\beta S^{*}\left(1+m S^{*}\right)}{\left(1+m S^{*}+n I^{*}\right)^{2}}+q b-\left(u+\alpha_{2}+\delta+\gamma\right)=-\frac{n \beta S^{*} I^{*}}{\left(1+m S^{*}+n I^{*}\right)^{2}}<0, \\
& a_{33}{ }^{*}=-\left(u+\alpha_{3}+\varepsilon\right)<0 .
\end{aligned}
$$

With regard to $E^{*}$, we have the following characteristic equation:

$$
\lambda^{3}+D \lambda^{2}+E \lambda+F=0
$$

where

$$
\begin{aligned}
D= & \frac{\beta I^{*}\left(1+n I^{*}+n S^{*}\right)}{\left(1+m S^{*}+n I^{*}\right)^{2}}+\left(u+\alpha_{3}+\varepsilon\right)+(u-b)>0, \\
E= & \frac{\beta I^{*}}{\left(1+m S^{*}+n I^{*}\right)^{2}}\left[\left(u+\alpha_{3}+\varepsilon\right)\left(1+n S^{*}+n I^{*}\right)-(b-u) n S^{*}\right. \\
& \left.+\left(u+\alpha_{2}+\delta-b\right)\left(1+n I^{*}\right)\right]-(b-u)\left(u+\alpha_{3}+\varepsilon\right) \\
> & 0
\end{aligned}
$$

$$
\begin{aligned}
F= & \frac{\beta I^{*}}{\left(1+m S^{*}+n I^{*}\right)^{2}}\left[\left(u+\alpha_{3}+\varepsilon\right)\left(u+\alpha_{2}+\delta-b\right)\left(1+n I^{*}\right) .\right. \\
& \left.+(u-b)\left(u+\alpha_{3}+\varepsilon\right) n S^{*}+\delta\left(u+\alpha_{3}-b\right)(1+n) I^{*}\right]
\end{aligned}
$$

$>0$.

Then we get

$$
\begin{aligned}
D E-F= & {\left[D_{1} E_{1}+(u-b) E_{1}+D_{1}(u-b)\left(u+\alpha_{3}+\varepsilon\right)+(u-b)\left(u+\alpha_{3}+\varepsilon\right)^{2} .\right.} \\
& \left.+(u-b)^{2}\left(u+\alpha_{3}+\varepsilon\right)\right]+\frac{\beta I^{*}}{\left(1+m S^{*}+n I^{*}\right)^{2}}\left[\delta(b+\varepsilon)\left(1+n I^{*}\right)\right. \\
& \left.+\left(u+\alpha_{3}+\varepsilon\right)^{2}\left(1+n S^{*}+n I^{*}\right)\right] \\
> & 0
\end{aligned}
$$


where

$$
\begin{aligned}
D_{1}= & \frac{\beta I^{*}\left(1+n I^{*}+n S^{*}\right)}{\left(1+m S^{*}+n I^{*}\right)^{2}}, \\
E_{1}= & \frac{\beta I^{*}}{\left(1+m S^{*}+n I^{*}\right)^{2}}\left[\left(u+\alpha_{3}+\varepsilon\right)\left(n S^{*}+n I^{*}+1\right)-(b-u) n S^{*}\right. \\
& \left.+\left(u+\alpha_{2}+\delta-b\right)\left(1+n I^{*}\right)\right] .
\end{aligned}
$$

Therefore, we can get $E^{*}$ is locally asymptotically stable when it exists. According to the proof of Theorem 2 in [45], the endemic equilibrium $E^{*}$ is globally asymptotically stable. This completes the proof of Theorem 3.1.

\section{The threshold of stochastic system (3)}

In the previous section, we discussed the threshold condition of deterministic system (2). In this section, we investigate the threshold conditions of disease extinction and persistence for stochastic SIQS system (3). First, we give the following definition [25].

\section{Definition 4.1}

(i) The disease $I(t)$ is said to be extinct if $\lim _{t \rightarrow+\infty} I(t)=0$;

(ii) The disease $I(t)$ is said to be permanent in mean if there is a positive constant $\phi$ such that $\lim _{t \rightarrow \infty} \inf \langle I(t)\rangle \geq \phi$.

Let

$$
\widetilde{R}=R_{0}-\frac{\sigma^{2} A^{2}}{2(A m+u-b)^{2}\left(u+\alpha_{2}+\delta+\gamma-q b\right)} .
$$

\subsection{Extinction}

Theorem 4.1 In system (3), let $(S(t), I(t), Q(t))$ be the solution for any initial value $(S(0), I(0), Q(0)) \in \Gamma$. If one of the following conditions is satisfied:

(i) $\sigma^{2}>\max \left\{\frac{\beta^{2}}{2\left(u+\alpha_{2}+\delta+\gamma-q b\right)}, \frac{\beta(A m+u-b)}{A}\right\}$,

(ii) $\widetilde{R}<1$ and $\sigma^{2}<\frac{\beta(A m+u-b)}{A}$,

then the infectious disease of system (3) goes to extinction a.s. Moreover, we have

$$
\begin{aligned}
& \lim _{t \rightarrow \infty} S(t)=\frac{A}{u-b} \quad \text { a.s. } \\
& \lim _{t \rightarrow \infty} Q(t)=0 \quad \text { a.s. }
\end{aligned}
$$

Proof Let $V_{3}=\ln I(t)$. Applying Itô's formula on $V_{3}$, we obtain

$$
d V_{3}=L V_{3} d t+\frac{\sigma S(t)}{1+m S(t)+n I(t)} d B(t)
$$

where

$$
L V_{3}=\frac{\beta S(t)}{1+m S(t)+n I(t)}+q b-\left(u+\alpha_{2}+\delta+\gamma\right)-\frac{\sigma^{2} S^{2}(t)}{2(1+m S(t)+n I(t))^{2}}
$$


Integrating both sides of (9) from 0 to $t$ yields

$$
\ln I(t)=\int_{0}^{t} L V_{3}(S(\xi), I(\xi)) d \xi+H(t)
$$

Let $H(t)=M(t)+\ln I(0)$, where $M(t)=\int_{0}^{t} \frac{\sigma S(\xi)}{1+m S(\xi)+n I(\xi)} d B(\xi)$ is a local continuous martingale with $M(0)=0$. Since $(S(t), I(t)) \in \Gamma$, then we have $\lim _{t \rightarrow \infty} \sup \frac{M(t)}{t}=0$ by the strong law of large numbers for martingales [46]. So we get $\lim _{t \rightarrow \infty} \sup \frac{H(t)}{t}=0$.

Case (i): Due to $\sigma^{2}>\frac{\beta(A m+u-b)}{A}$, we can obtain

$$
\begin{aligned}
L V_{3}(S(t), I(t))= & \frac{\beta S(t)}{1+m S(t)+n I(t)}+q b-\left(u+\alpha_{2}+\delta+\gamma\right) \\
& -\frac{\sigma^{2} S^{2}(t)}{2(1+m S(t)+n I(t))^{2}} \\
= & -\frac{\sigma^{2}}{2}\left(\frac{S(t)}{1+m S(t)+n I(t)}-\frac{\beta}{\sigma^{2}}\right)^{2}+\frac{\beta^{2}}{2 \sigma^{2}}+q b \\
& -\left(u+\alpha_{2}+\delta+\gamma\right) \\
\leq & \frac{\beta^{2}}{2 \sigma^{2}}+q b-\left(u+\alpha_{2}+\delta+\gamma\right) .
\end{aligned}
$$

In view of (11),

$$
\ln I(t) \leq \int_{0}^{t}\left[\frac{\beta^{2}}{2 \sigma^{2}}+q b-\left(u+\alpha_{2}+\delta+\gamma\right)\right] d \xi+H(t)
$$

Dividing both sides of (12) by $t>0$, we have

$$
\frac{\ln I(t)}{t} \leq \frac{\beta^{2}}{2 \sigma^{2}}+q b-\left(u+\alpha_{2}+\delta+\gamma\right)+\frac{H(t)}{t} .
$$

Then, taking the limit superior on both sides of (13), when $\sigma^{2}>\frac{\beta^{2}}{2\left(u+\alpha_{2}+\delta+\gamma-q b\right)}$, we gain

$$
\lim _{t \rightarrow \infty} \sup \frac{\ln I(t)}{t} \leq \frac{\beta^{2}}{2 \sigma^{2}}+q b-\left(u+\alpha_{2}+\delta+\gamma\right)<0
$$

That implies $\lim _{t \rightarrow \infty} I(t)=0$ and the infectious disease goes to extinction.

Case (ii): In this case, since $S \in \Gamma$, we have

$$
\begin{aligned}
L V_{3}(S(t), I(t))= & -\frac{\sigma^{2}}{2}\left(\frac{S(t)}{1+m S(t)+n I(t)}-\frac{\beta}{\sigma^{2}}\right)^{2}+\frac{\beta^{2}}{2 \sigma^{2}}+q b \\
& -\left(u+\alpha_{2}+\delta+\gamma\right) \\
\leq & -\frac{\sigma^{2}}{2}\left(\frac{\frac{A}{u-b}}{1+m \frac{A}{u-b}}-\frac{\beta}{\sigma^{2}}\right)^{2}+\frac{\beta^{2}}{2 \sigma^{2}}+q b \\
& -\left(u+\alpha_{2}+\delta+\gamma\right) \\
= & \frac{A \beta}{(A m+u-b)}-\frac{A^{2} \sigma^{2}}{2(A m+u-b)^{2}}+q b \\
& -\left(u+\alpha_{2}+\delta+\gamma\right)
\end{aligned}
$$




$$
=\left(u+\alpha_{2}+\delta+\gamma-q b\right)(\widetilde{R}-1) \text {. }
$$

From (11) we obtain

$$
\ln I(t) \leq \int_{0}^{t}\left(u+\alpha_{2}+\delta+\gamma-q b\right)(\widetilde{R}-1) d \xi+H(t)
$$

Dividing both sides of (15) by $t>0$ yields

$$
\frac{\ln I(t)}{t} \leq\left(u+\alpha_{2}+\delta+\gamma-q b\right)(\widetilde{R}-1)+\frac{H(t)}{t} .
$$

Taking the superior limit on both sides of (16), when $\widetilde{R}<1$, we have

$$
\lim _{t \rightarrow \infty} \sup \frac{\ln I(t)}{t} \leq\left(u+\alpha_{2}+\delta+\gamma-q b\right)(\widetilde{R}-1)
$$

The condition $\widetilde{R}<1$ implies that $\lim _{t \rightarrow \infty} I(t)=0$.

Next, solving the third equation of system (3), one can obtain that

$$
Q(t)=e^{-\left(u+\alpha_{3}+\varepsilon\right) t}\left[Q(0)+\int_{0}^{t} \delta I(r) e^{\left(u+\alpha_{3}+\varepsilon\right) r} d r\right]
$$

Applying L'Hospital's rule, we have

$$
\lim _{t \rightarrow \infty} Q(t)=\lim _{t \rightarrow \infty} \frac{Q(0)+\int_{0}^{t} \delta I(r) e^{\left(u+\alpha_{3}+\varepsilon\right) r} d r}{e^{\left(u+\alpha_{3}+\varepsilon\right) t}}=\lim _{t \rightarrow \infty} \frac{\delta I(t)}{u+\alpha_{3}+\varepsilon}=0 \quad \text { a.s. }
$$

According to system (3), we obtain

$$
d(S(t)+I(t)+Q(t))=\left[A-(u-b)(S(t)+I(t)+Q(t))-\alpha_{2} I(t)-\alpha_{3} Q(t)\right] d t
$$

Then

$$
\begin{aligned}
& S(t)+I(t)+Q(t) \\
& \quad=e^{-(u-b) t}\left\{[S(0)+I(0)+Q(0)]+\int_{0}^{t}\left[A-\alpha_{2} I(r)-\alpha_{3} Q(r)\right] e^{(u-b) r} d r\right\} .
\end{aligned}
$$

Similarly, one can get that

$$
\lim _{t \rightarrow \infty} S(t)=\lim _{t \rightarrow \infty} \frac{A-\alpha_{2} I(r)-\alpha_{3} Q(r)}{u-b}=\frac{A}{u-b} \quad \text { a.s. }
$$

This proof is completed.

\subsection{Permanence in mean}

Theorem 4.2 If $\widetilde{R}>1$, then the infectious disease is permanent in mean, and

$$
\lim _{t \rightarrow \infty} \inf \langle I(t)\rangle \geq I_{*}
$$




$$
\begin{aligned}
& \lim _{t \rightarrow \infty} \inf \left\langle\frac{A}{u-b}-S(t)\right\rangle \geq\left[1+\frac{\alpha_{2}}{u-b}+\frac{\delta\left(u+\alpha_{3}-b\right)}{(u-b)\left(u+\alpha_{3}+\varepsilon\right)}\right] I_{*}, \\
& \lim _{t \rightarrow \infty} \inf \langle Q(t)\rangle \geq \frac{\delta}{u+\alpha_{3}+\varepsilon} I_{*},
\end{aligned}
$$

where

$$
I_{*}=\frac{(A m+u-b)\left(u+\alpha_{2}+\delta+\gamma-q b\right)}{\beta\left[(u-b)+\alpha_{2}+\frac{\delta\left(u+\alpha_{3}-b\right)}{\left(u+\alpha_{3}+\varepsilon\right)}\right]+n(u-b)\left(u+\alpha_{2}+\delta+\gamma-q b\right)}(\widetilde{R}-1) .
$$

Proof Through system (3), we have

$$
\begin{aligned}
& d\left(S(t)+I(t)+\frac{\varepsilon+b}{u+\alpha_{3}+\varepsilon} Q(t)\right) d t \\
& \quad=\left\{A-(u-b) S(t)-\left[u+\alpha_{2}-b+\frac{\delta\left(u+\alpha_{3}-b\right)}{u+\alpha_{3}+\varepsilon}\right] I(t)\right\} d t .
\end{aligned}
$$

Integrating from 0 to $t$ and dividing by $t$ on both sides of (18), one can get

$$
\begin{aligned}
\Theta(t) & =\frac{S(t)-S(0)}{t}+\frac{I(t)-I(0)}{t}+\frac{\varepsilon+b}{u+\alpha_{3}+\varepsilon} \frac{Q(t)-Q(0)}{t} \\
& =A-(u-b)\langle S(t)\rangle-\left[u+\alpha_{2}-b+\frac{\delta\left(u+\alpha_{3}-b\right)}{u+\alpha_{3}+\varepsilon}\right]\langle I(t)\rangle .
\end{aligned}
$$

Therefore, we obtain that

$$
\langle S(t)\rangle=\frac{A}{u-b}-\left[1+\frac{\alpha_{2}}{u-b}+\frac{\delta\left(u+\alpha_{3}-b\right)}{(u-b)\left(u+\alpha_{3}+\varepsilon\right)}\right]\langle I(t)\rangle-\frac{\Theta(t)}{u-b} .
$$

Applying Itô's formula, we get

$$
\begin{aligned}
d[ & \left.\left(1+m \frac{A}{u-b}\right) \ln I(t)+n I(t)\right] \\
= & {\left[\frac{\left(1+m \frac{A}{u-b}\right) \beta S(t)}{1+m S(t)+n I(t)}-\left(1+m \frac{A}{u-b}\right)\left(u+\alpha_{2}+\delta+\gamma-q b\right)\right.} \\
& +\frac{n \beta S(t) I(t)}{1+m S(t)+n I(t)}-n I(t)\left(u+\alpha_{2}+\delta+\gamma-q b\right) \\
& \left.-\frac{1}{2} \frac{\left(1+m \frac{A}{u-b}\right) \sigma^{2} S^{2}(t)}{(1+m S(t)+n I(t))^{2}}\right] d t \\
& +\left[\frac{\left(1+m \frac{A}{u-b}\right) \sigma S(t)}{1+m S(t)+n I(t)}+\frac{n \sigma S(t) I(t)}{1+m S(t)+n I(t)}\right] d B(t) \\
\geq & {\left[\beta S(t)-\left(1+m \frac{A}{u-b}\right)\left(u+\alpha_{2}+\delta+\gamma-q b\right)\right.} \\
& \left.-n\left(u+\alpha_{2}+\delta+\gamma-q b\right) I(t)-\frac{\sigma^{2}\left(\frac{A}{u-b}\right)^{2}}{2\left(1+m \frac{A}{u-b}\right)}\right] d t \\
& +\left[\frac{\left(1+m \frac{A}{u-b}\right) \sigma S(t)}{1+m S(t)+n I(t)}+\frac{n \sigma S(t) I(t)}{1+m S(t)+n I(t)}\right] d B(t) .
\end{aligned}
$$


Integrating from 0 to $t$ and dividing by $t$ on both sides of (21), one can obtain that

$$
\begin{aligned}
(1+ & \left.m \frac{A}{u-b}\right) \frac{\ln I(t)-\ln I(0)}{t}+n \frac{I(t)-I(0)}{t} \\
\geq & \beta\langle S(t)\rangle-\left(1+m \frac{A}{u-b}\right)\left(u+\alpha_{2}+\delta+\gamma-q b\right) \\
& -n\left(u+\alpha_{2}+\delta+\gamma-q b\right)\langle I(t)\rangle-\frac{\sigma^{2}\left(\frac{A}{u-b}\right)^{2}}{2\left(1+m \frac{A}{u-b}\right)}+\frac{M_{1}(t)}{t} \\
= & \left(1+m \frac{A}{u-b}\right)\left(u+\alpha_{2}+\delta+\gamma-q b\right)(\widetilde{R}-1) \\
& -\left[\beta+\frac{\beta \alpha_{2}}{u-b}+\frac{\beta \delta\left(u+\alpha_{3}-b\right)}{(u-b)\left(u+\alpha_{3}+\varepsilon\right)}+n\left(u+\alpha_{2}+\delta+\gamma-q b\right)\right]\langle I(t)\rangle \\
& -\frac{\beta \Theta(t)}{u-b}+\frac{M_{1}(t)}{t},
\end{aligned}
$$

where

$$
M_{1}(t)=\int_{0}^{t}\left[\frac{\left(1+m \frac{A}{u-b}\right) \sigma S(\xi)}{1+m S(\xi)+n I(\xi)}+\frac{n \sigma S(\xi) I(\xi)}{1+m S(\xi)+n I(\xi)}\right] d B(\xi)
$$

Then, we can calculate from (22)

$$
\begin{aligned}
\ln I(t) \geq & F(t)-\frac{\beta+\frac{\beta \alpha_{2}}{u-b}+\frac{\beta \delta\left(u+\alpha_{3}-b\right)}{(u-b)\left(u+\alpha_{3}+\varepsilon\right)} n\left(u+\alpha_{2}+\delta+\gamma-q b\right)}{1+m \frac{A}{u-b}} \int_{0}^{t} I(s) d s \\
& +\left[\left(u+\alpha_{2}+\delta+\gamma-q b\right)(\widetilde{R}-1)\right] t,
\end{aligned}
$$

where

$$
F(t)=-\frac{\beta t \Theta(t)}{A m+u-b}+\frac{M_{1}(t)}{1+m \frac{A}{u-b}}-\frac{n}{1+m \frac{A}{u-b}}[I(t)-I(0)]+\ln I(0) .
$$

Since $S(t)+I(t)+Q(t) \leq \frac{A}{u-b}$, then $\lim _{t \rightarrow \infty} \frac{S(t)}{t}=\lim _{t \rightarrow \infty} \frac{I(t)}{t}=\lim _{t \rightarrow \infty} \frac{Q(t)}{t}=0$ and $\lim _{t \rightarrow \infty} \Theta(t)=0$. Utilizing the strong law of large numbers of martingales, we have $\lim _{t \rightarrow \infty} \frac{F(t)}{t}=0$.

Taking the inferior limit of both sides of (23) and using Lemma 2.4 in [47], we obtain

$$
\lim _{t \rightarrow \infty} \inf |I(t)\rangle \geq \frac{\left(1+m \frac{A}{u-b}\right)\left(u+\alpha_{2}+\delta+\gamma-q b\right)}{\beta+\frac{\beta \alpha_{2}}{u-b}+\frac{\beta \delta\left(u+\alpha_{3}-b\right)}{(u-b)\left(u+\alpha_{3}+\varepsilon\right)}+n\left(u+\alpha_{2}+\delta+\gamma-q b\right)}(\widetilde{R}-1) .
$$

According to (20), one can obtain that

$$
\lim _{t \rightarrow \infty} \inf \left\langle\frac{A}{u-b}-S(t)\right\rangle \geq\left[1+\frac{\alpha_{2}}{u-b}+\frac{\delta\left(u+\alpha_{3}-b\right)}{(u-b)\left(u+\alpha_{3}+\varepsilon\right)}\right] I_{*} .
$$

Integrating from 0 to $t$ and dividing by $t$ on both sides of the last equation of system (3) yields

$$
\frac{Q(t)-Q(0)}{t}=\delta\langle I(t)\rangle-\left(u+\alpha_{3}+\varepsilon\right)\langle Q(t)\rangle .
$$


Therefore, we have

$$
\lim _{t \rightarrow \infty} \inf \langle Q(t)\rangle \geq \frac{\delta}{u+\alpha_{3}+\varepsilon} I_{*}
$$

This finishes the proof of Theorem 4.2.

\section{Asymptotic behavior of system (3)}

According to Theorem 3.1, if $R_{0}>1$, there exists a unique endemic equilibrium $E^{*}$ of deterministic system (2) and it is globally asymptotically stable. However, due to the interference of white noise, there is no equilibrium for system (3). It is interesting to discuss how the solution of stochastic system (3) oscillates near $E^{*}$. Next, we prove that when the intensity of the white noise is small, the solution of stochastic system (3) will oscillate slightly around $E^{*}$. That is, the infectious disease is persistent.

Let

$$
\begin{aligned}
& \Delta_{1}=(u-b)\left(1+c_{1}\right)-\frac{c_{3} I^{*} \sigma^{2}}{2}, \\
& \Delta_{2}=\left(u+\alpha_{2}-b\right)+c_{1}\left(u+\alpha_{2}+\delta-b\right), \\
& \Delta_{3}=\left(u+\alpha_{3}-b\right)+c_{2}\left(u+\alpha_{3}+\varepsilon\right) .
\end{aligned}
$$

Theorem 5.1 If the following conditions are met:

(i) $\widetilde{R}>1$,

(ii) $\sigma^{2}<\frac{2(u-b)\left(1+c_{1}\right)}{c_{3} I^{*}}$,

then the solution $(S(t), I(t), Q(t))$ of system (3) with initial value $(S(0), I(0), Q(0)) \in R_{+}^{3}$ has the nature

$$
\lim _{t \rightarrow \infty} \sup \frac{1}{t} E \int_{0}^{t}\left[\left(S-M_{S} S^{*}\right)^{2}+\left(I-I^{*}\right)^{2}+\left(Q-Q^{*}\right)^{2}\right] d \xi \leq \frac{\theta}{\varphi}
$$

where

$$
\begin{aligned}
& M_{S}=\frac{2(u-b)\left(1+c_{1}\right)}{2(u-b)\left(1+c_{1}\right)-c_{3} I^{*} \sigma^{2}}, \\
& \theta=\frac{c_{3} I^{*}(u-b)\left(1+c_{1}\right) \sigma^{2} S^{* 2}}{2(u-b)\left(1+c_{1}\right)-c_{3} I^{*} \sigma^{2}}, \\
& \varphi=\min \left\{\Delta_{1}, \Delta_{2}, \Delta_{3}\right\},
\end{aligned}
$$

and $c_{1}, c_{2}, c_{3}$ are positive constants, we give the values in the following proof.

Proof Because of $\widetilde{R}>1$, then $R_{0}>1$, system (2) has a unique endemic equilibrium satisfying the following equation:

$$
\begin{aligned}
& A=u S^{*}+\frac{\beta I^{*} S^{*}}{1+m S^{*}+n I^{*}}-b\left(S^{*}+Q^{*}\right)-(1-q) b I^{*}-\gamma I^{*}-\varepsilon Q^{*}, \\
& \frac{\beta S^{*}}{1+m S^{*}+n I^{*}}=u+\alpha_{2}+\delta+\gamma-q b, \\
& \delta I^{*}=\left(u+\alpha_{3}+\varepsilon\right) Q^{*} .
\end{aligned}
$$


Let

$$
\begin{aligned}
\widetilde{V}= & \frac{1}{2}\left(S-S^{*}+I-I^{*}+Q-Q^{*}\right)^{2}+\frac{c_{1}}{2}\left(S-S^{*}+I-I^{*}\right)^{2}+\frac{c_{2}}{2}\left(Q-Q^{*}\right)^{2} \\
& +c_{3}\left(I-I^{*}-I^{*} \ln \frac{I}{I^{*}}\right) \\
= & \widetilde{V}_{1}+c_{1} \widetilde{V}_{2}+c_{2} \widetilde{V}_{3}+c_{3} \widetilde{V}_{4},
\end{aligned}
$$

where $\widetilde{V}_{1}=\frac{1}{2}\left(S-S^{*}+I-I^{*}+Q-Q^{*}\right)^{2}, \widetilde{V}_{2}=\frac{1}{2}\left(S-S^{*}+I-I^{*}\right)^{2}, \widetilde{V}_{3}=\frac{1}{2}\left(Q-Q^{*}\right)^{2}$, and $\widetilde{V}_{4}=\left(I-I^{*}-I^{*} \ln \frac{I}{I^{*}}\right)$. Applying Itô’'s formula, we obtain

$$
\begin{aligned}
L \widetilde{V}_{1}= & \left(S-S^{*}+I-I^{*}+Q-Q^{*}\right)\left[A-(u-b) S-\left(u+\alpha_{2}-b\right) I-\left(u+\alpha_{3}-b\right) Q\right] \\
= & -(u-b)\left(S-S^{*}\right)^{2}-\left(u+\alpha_{2}-b\right)\left(I-I^{*}\right)^{2}-\left(u+\alpha_{3}-b\right)\left(Q-Q^{*}\right)^{2} \\
& -\left(2 u+\alpha_{2}-2 b\right)\left(S-S^{*}\right)\left(I-I^{*}\right)-\left(2 u+\alpha_{2}+\alpha_{3}-2 b\right)\left(I-I^{*}\right)\left(Q-Q^{*}\right) \\
& -\left(2 u+\alpha_{3}-2 b\right)\left(S-S^{*}\right)\left(Q-Q^{*}\right), \\
L \widetilde{V}_{2}= & \left(S-S^{*}+I-I^{*}\right)\left[A-(u-b) S-\left(u+\alpha_{2}+\delta-b\right) I+(\varepsilon+b) Q\right] \\
= & -(u-b)\left(S-S^{*}\right)^{2}-\left(u+\alpha_{2}+\delta-b\right)\left(I-I^{*}\right)^{2}+(\varepsilon+b)\left(I-I^{*}\right)\left(Q-Q^{*}\right) \\
& -\left(2 u+\alpha_{2}+\delta-2 b\right)\left(S-S^{*}\right)\left(I-I^{*}\right)+(\varepsilon+b)\left(S-S^{*}\right)\left(Q-Q^{*}\right), \\
L \widetilde{V}_{3}= & \left(Q-Q^{*}\right)\left[\delta I-\left(u+\alpha_{3}+\varepsilon\right) Q\right] \\
= & \delta\left(Q-Q^{*}\right)\left(I-I^{*}\right)-\left(u+\alpha_{3}+\varepsilon\right)\left(Q-Q^{*}\right)^{2}, \\
L \widetilde{V}_{4}= & \left(I-I^{*}\right)\left[\frac{\beta S}{(1+m S+n I)}-\left(u+\alpha_{2}+\delta+\gamma-q b\right)\right]+\frac{I^{*} \sigma^{2} S^{2}}{2(1+m S+n I)^{2}} \\
= & \left(I-I^{*}\right) S\left(\frac{\beta}{1+m S+n I}-\frac{\beta}{1+m S^{*}+n I^{*}}\right) \\
& +\frac{\beta}{1+m S^{*}+n I^{*}}\left(I-I^{*}\right)\left(S-S^{*}\right)+\frac{I^{*} \sigma^{2} S^{2}}{2(1+m S+n I)^{2}} \\
\leq & \frac{\beta}{1+m S^{*}+n I^{*}}\left(I-I^{*}\right)\left(S-S^{*}\right)+\frac{I^{*} \sigma^{2} S^{2}}{2(1+m S+n I)^{2}} .
\end{aligned}
$$

Thus, we have

$$
\begin{aligned}
L \widetilde{V} \leq & -(u-b)\left(1+c_{1}\right)\left(S-S^{*}\right)^{2}-\left[\left(u+\alpha_{2}-b\right)+c_{1}\left(u+\alpha_{2}+\delta-b\right)\right]\left(I-I^{*}\right)^{2} \\
& -\left[\left(u+\alpha_{3}-b\right)+c_{2}\left(u+\alpha_{3}+\varepsilon\right)\right]\left(Q-Q^{*}\right)^{2}-\left[\left(2 u+\alpha_{2}-2 b\right)\right. \\
& \left.+c_{1}\left(2 u+\alpha_{2}+\delta-2 b\right)\right]\left(S-S^{*}\right)\left(I-I^{*}\right)-\left[\left(2 u+\alpha_{2}+\alpha_{3}-2 b\right)-c_{2} \delta\right. \\
& \left.-c_{1}(b+\varepsilon)\right]\left(I-I^{*}\right)\left(Q-Q^{*}\right)-\left[\left(2 u+\alpha_{3}-2 b\right)-c_{1}(b+\varepsilon)\right]\left(S-S^{*}\right)\left(Q-Q^{*}\right) \\
& +c_{3}\left[\frac{\beta}{1+m S^{*}+n I^{*}}\left(I-I^{*}\right)\left(S-S^{*}\right)+\frac{I^{*} \sigma^{2} S^{2}}{2(1+m S+n I)^{2}}\right] .
\end{aligned}
$$


Next, we choose

$$
\begin{aligned}
& c_{1}=\frac{2 u+\alpha_{3}-2 b}{\varepsilon+b}, \\
& c_{2}=\frac{\alpha_{2}}{\delta} \\
& c_{3}=\frac{\left[(\varepsilon+b)\left(2 u+\alpha_{2}-2 b\right)+\left(2 u+\alpha_{3}-2 b\right)\left(2 u+\alpha_{2}+\delta-2 b\right)\right]\left(1+m S^{*}+n I^{*}\right)}{\beta(\varepsilon+b)},
\end{aligned}
$$

then

$$
\begin{aligned}
L \widetilde{V} \leq & -(u-b)\left(1+c_{1}\right)\left(S-S^{*}\right)^{2}-\left[\left(u+\alpha_{3}-b\right)+c_{2}\left(u+\alpha_{3}+\varepsilon\right)\right]\left(Q-Q^{*}\right)^{2} \\
& -\left[\left(u+\alpha_{2}-b\right)+c_{1}\left(u+\alpha_{2}+\delta-b\right)\right]\left(I-I^{*}\right)^{2}+c_{3} \frac{I^{*} \sigma^{2} S^{2}}{2(1+m S+n I)^{2}} \\
\leq & -\left[(u-b)\left(1+c_{1}\right)-\frac{c_{3} I^{*} \sigma^{2}}{2}\right]\left[S-\frac{2(u-b)\left(1+c_{1}\right)}{2(u-b)\left(1+c_{1}\right)-c_{3} I^{*} \sigma^{2}} S^{*}\right]^{2} \\
& -\left[\left(u+\alpha_{2}-b\right)+c_{1}\left(u+\alpha_{2}+\delta-b\right)\right]\left(I-I^{*}\right)^{2}-\left[\left(u+\alpha_{3}-b\right)\right. \\
& \left.+c_{2}\left(u+\alpha_{3}+\varepsilon\right)\right]\left(Q-Q^{*}\right)^{2}+\frac{c_{3} I^{*}(u-b)\left(1+c_{1}\right) \sigma^{2} S^{* 2}}{2(u-b)\left(1+c_{1}\right)-c_{3} I^{*} \sigma^{2}} \\
= & -\Delta_{1}\left[S-\frac{2(u-b)\left(1+c_{1}\right)}{2(u-b)\left(1+c_{1}\right)-c_{3} I^{*} \sigma^{2}} S^{*}\right]^{2}-\Delta_{2}\left(I-I^{*}\right)^{2} \\
& -\Delta_{3}\left(Q-Q^{*}\right)^{2}+\theta .
\end{aligned}
$$

Here, $\Delta_{1}=(u-b)\left(1+c_{1}\right)-\frac{c_{3} I^{*} \sigma^{2}}{2}, \Delta_{2}=\left(u+\alpha_{2}-b\right)+c_{1}\left(u+\alpha_{2}+\delta-b\right), \Delta_{3}=\left(u+\alpha_{3}-b\right)+$ $c_{2}\left(u+\alpha_{3}+\varepsilon\right)$, and $\theta=\frac{c_{3} I^{*}(u-b)\left(1+c_{1}\right) \sigma^{2} S^{* 2}}{2(u-b)\left(1+c_{1}\right)-c_{3} I^{*} \sigma^{2}}$. Furthermore, we achieve that

$$
\begin{aligned}
d \widetilde{V} \leq & \left\{-\Delta_{1}\left[S-\frac{2(u-b)\left(1+c_{1}\right)}{2(u-b)\left(1+c_{1}\right)-c_{3} I^{*} \sigma^{2}} S^{*}\right]^{2}-\Delta_{2}\left(I-I^{*}\right)^{2}\right. \\
& \left.-\Delta_{3}\left(Q-Q^{*}\right)^{2}+\theta\right\} d t+\left(I-I^{*}\right) \frac{\sigma S}{1+m S+n I} d B(t) .
\end{aligned}
$$

Integrating the two sides of (28) from 0 to $t$ leads to

$$
\begin{aligned}
& \widetilde{V}(S(t), I(t), Q(t))-\widetilde{V}(S(0), I(0), Q(0)) \\
& \leq \int_{0}^{t}\left\{-\Delta_{1}\left[S(\xi)-\frac{2(u-b)\left(1+c_{1}\right)}{2(u-b)\left(1+c_{1}\right)-c_{3} I^{*} \sigma^{2}} S^{*}\right]^{2}-\Delta_{2}\left(I(\xi)-I^{*}\right)^{2}\right. \\
& \left.\quad-\Delta_{3}\left(Q(\xi)-Q^{*}\right)^{2}+\theta\right\} d \xi \\
& \quad+\int_{0}^{t}\left(I(\xi)-I^{*}\right) \frac{\sigma S(\xi)}{1+m S(\xi)+n I(\xi)} d B(\xi)
\end{aligned}
$$


where $\int_{0}^{t}\left(I-I^{*}\right) \frac{\sigma S I}{1+m S+n I} d B(\xi)$ is the local continuous martingale. And now, taking expectations of (29), we have

$$
\begin{aligned}
& E \tilde{V}(S(t), I(t), Q(t))-E \tilde{V}(S(0), I(0), Q(0)) \\
& \quad \leq E \int_{0}^{t}\left\{-\Delta_{1}\left[S(\xi)-\frac{2(u-b)\left(1+c_{1}\right)}{2(u-b)\left(1+c_{1}\right)-c_{3} I^{*} \sigma^{2}} S^{*}\right]^{2}-\Delta_{2}\left(I(\xi)-I^{*}\right)^{2}\right. \\
& \left.\quad-\Delta_{3}\left(Q(\xi)-Q^{*}\right)^{2}\right\} d \xi+\theta t .
\end{aligned}
$$

Set $\varphi=\min \left\{\Delta_{1}, \Delta_{2}, \Delta_{3}\right\}$, then we can obtain

$$
\lim _{t \rightarrow \infty} \sup \frac{1}{t} E \int_{0}^{t}\left[\left(S-M_{S} S^{*}\right)^{2}+\left(I-I^{*}\right)^{2}+\left(Q-Q^{*}\right)^{2}\right] d \xi \leq \frac{\theta}{\varphi},
$$

where $M_{S}=\frac{2(u-b)\left(1+c_{1}\right)}{2(u-b)\left(1+c_{1}\right)-c_{3} I^{*} \sigma^{2}}$. This achieves the proof of Theorem 5.1.

\section{Conclusion and simulations}

In this paper, a stochastic SIQS epidemic model with vertical transmission and Beddington-DeAngelis incidence is constructed. Under the condition of ignoring random effects, the stability of disease-free equilibrium and endemic equilibrium of the corresponding deterministic system (2) is discussed. If $R_{0}<1$, the infectious disease of system (2) goes to extinction. If $R_{0}>1$, a unique endemic equilibrium is globally asymptotically stable. It indicates that the disease will prevail and persist in a population.

For stochastic system (3), we obtain threshold conditions for disease extinction and permanence in mean. If $\widetilde{R}<1$, the infectious disease of system (3) dies out. If $\widetilde{R}>1$, the infectious disease is permanent in mean and oscillates near the positive equilibrium $E^{*}=\left(S^{*}, I^{*}, Q^{*}\right)$. Because of $\widetilde{R}<R_{0}$, as a result, the larger environmental noise is favorable for the control of infectious disease.

In order to verify the above theoretical results, we use Euler-Maruyama (EM) method [48] to carry out computer simulations. In our numerical simulations, the related parameters of system (2) and system (3) are as follows:

$$
\begin{aligned}
& u=0.32, \quad \delta=0.58, \quad \beta=0.3, \quad b=0.2, \\
& q=0.26, \quad \varepsilon=0.32, \quad \alpha_{2}=0.12, \quad \alpha_{3}=0.23, \\
& \gamma=0.25, \quad m=0.01, \quad n=0.5 \text {. }
\end{aligned}
$$

If we select $A=0.48$, we get $R_{0}=0.9473<1$ by a simple calculation. It satisfies the first condition of Theorem 3.1, then system (2) has a disease-free equilibrium $E_{0}=(4,0,0)$ (see Fig. 1). On the other hand, set $A=0.72$, we compute $R_{0}=1.3942>1$ and gain the positive equilibrium $E^{*}=(4.8492,0.2918,0.1945)$ (see Fig. 2).

The coefficient is the same as Fig. 2, and select different $\sigma$. In the first place, set $\sigma=0.6$, $0.36=\sigma^{2}>\max \left\{\frac{\beta^{2}}{2\left(u+\alpha_{2}+\delta+\gamma-q b\right)}, \frac{\beta(A m+u-b)}{A}\right\}=\max \{0.078,0.0369\}=0.078$, this indicates that the disease can die out (see Fig. 3). Secondly, we have decreased the value of $\sigma$ to 0.21 , we can easily figure out $\widetilde{R}=0.8141<1$ and $0.0441=\sigma^{2}<\frac{\beta(A m+u-b)}{A}=0.053$. On the basis of Theorem 4.1, we can get the disease go extinct (see Fig. 4). In addition, we let $\sigma=0.055$ and gain $\widetilde{R}=1.3544>1$, which shows the disease is persistent (see Fig. 5) by Theorem 4.2. 


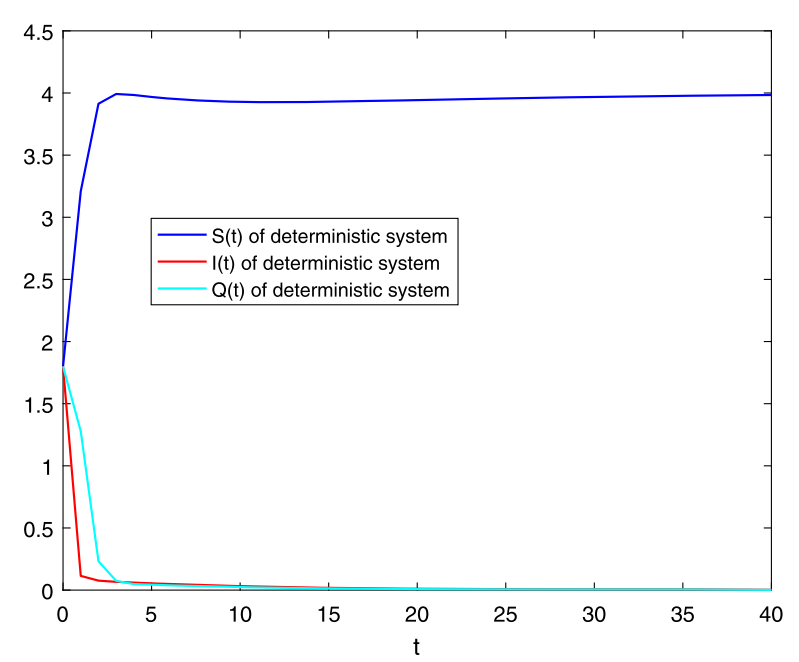

Figure 1 Time evolutions of deterministic system (2) with parameters $u=0.32, \delta=0.58, \beta=0.3, b=0.2$, $q=0.26, \varepsilon=0.32, \alpha_{2}=0.12, \alpha_{3}=0.23, \gamma=0.25, m=0.01, n=0.5, A=0.48$, where $R_{0}=0.9473<1$

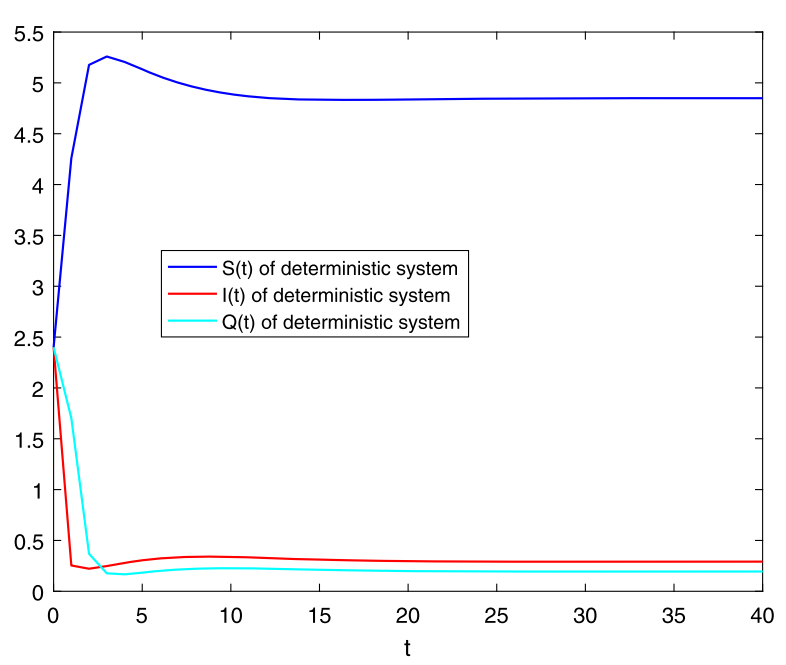

Figure 2 Time evolutions of deterministic system (2) with parameters $u=0.32, \delta=0.58, \beta=0.3, b=0.2$, $q=0.26, \varepsilon=0.32, \alpha_{2}=0.12, \alpha_{3}=0.23, \gamma=0.25, m=0.01, n=0.5, A=0.72$, where $R_{0}=1.3942>1$

In order to verify Theorem 5.1, we select another set of data for numerical simulations. We choose

$$
\begin{array}{ll}
u=0.4, & \delta=0.15, \quad A=1, \quad b=0.1, \quad q=0.3, \\
\varepsilon=0.35, \quad \beta=0.5, \quad \alpha_{2}=0.25, \quad \alpha_{3}=0.15, \\
\gamma=0.2, \quad m=0.01, \quad n=0.1, \quad \sigma=0.2 .
\end{array}
$$

From the conditions of Theorem 5.1, $\widetilde{R}=1.4482>1$, the endemic equilibrium is $E^{*}=$ $(2.0959,0.594,0.099), c_{1}=1.6667, c_{2}=2.5$, and $c_{3}=5.4378$. Therefore, we get $0.04=\sigma^{2}<$ $\frac{2(u-b)\left(1+c_{1}\right)}{c_{3} I^{*}}=0.4954$, which satisfies the second prerequisite of Theorem 5.1. We can see that the solution in (3) is oscillates around the equilibrium of the disease (see Fig. 6). 
(a)

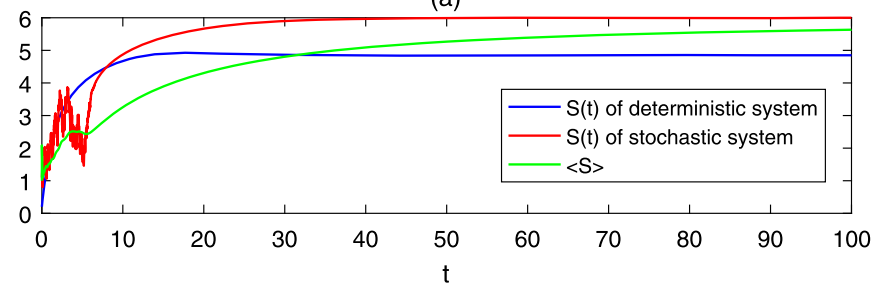

(b)

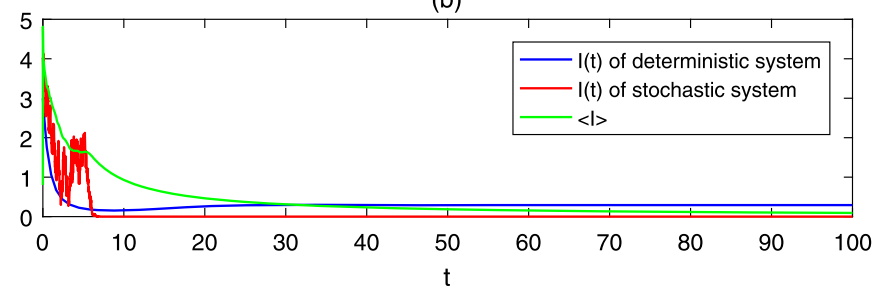

(c)

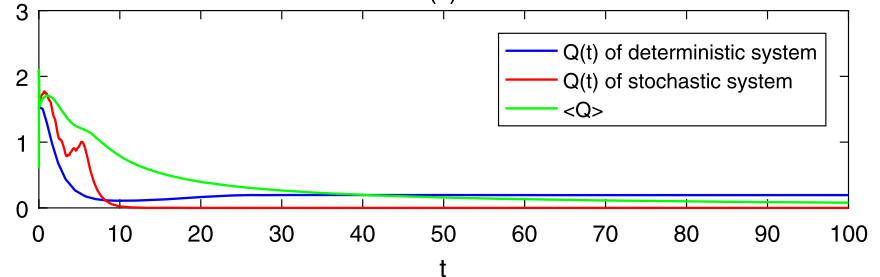

Figure 3 Comparison of system (2) and system (3), $u=0.32, \delta=0.58, \beta=0.3, b=0.2, q=0.26, \varepsilon=0.32$, $\alpha_{2}=0.12, \alpha_{3}=0.23, \gamma=0.25, m=0.01, n=0.5, A=0.72, \sigma=0.6$, where $R_{0}=1.3942>1$

(a)

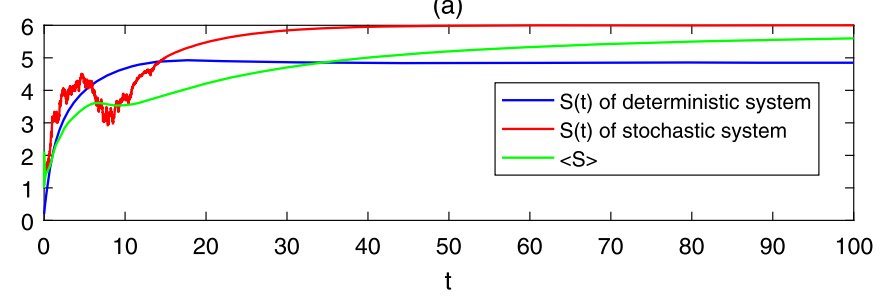

(b)

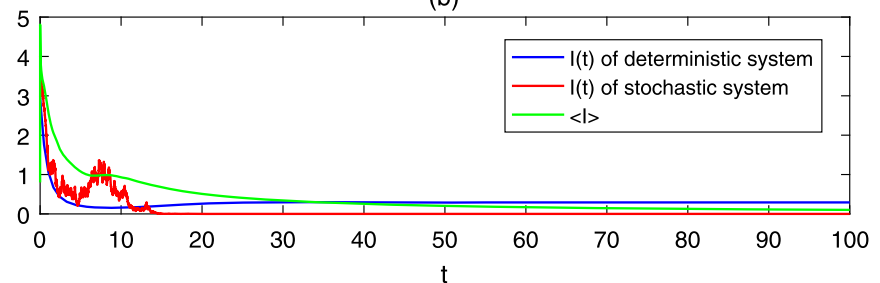

(c)

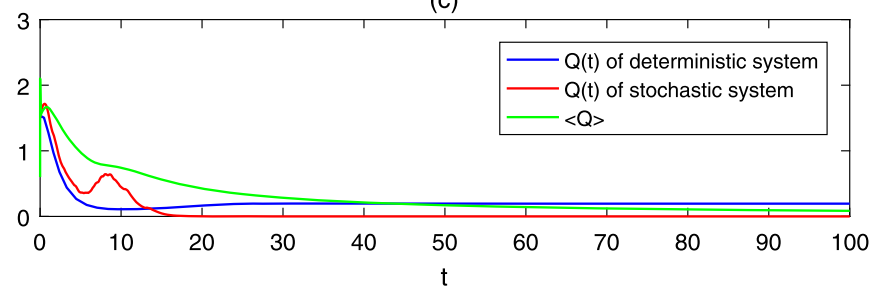

Figure 4 Comparison of system (2) and system (3), $u=0.32, \delta=0.58, \beta=0.3, b=0.2, q=0.26, \varepsilon=0.32$, $\alpha_{2}=0.12, \alpha_{3}=0.23, \gamma=0.25, m=0.01, n=0.5, A=0.72, \sigma=0.21$, where $R_{0}=1.3942>1, \widetilde{R}=0.8141<1$ 
(a)

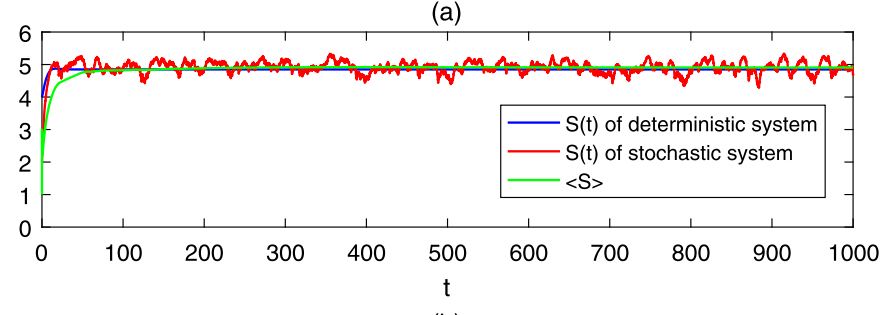

(b)

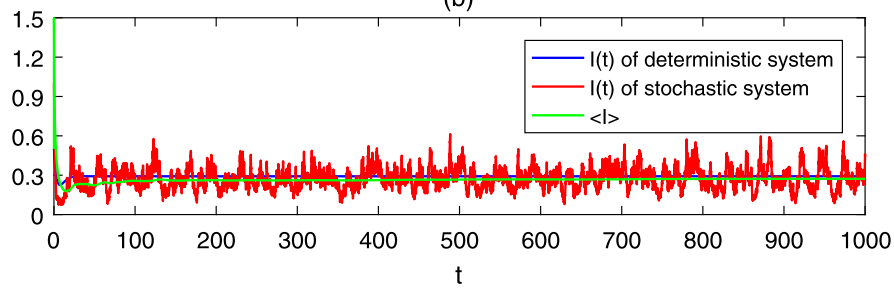

(c)

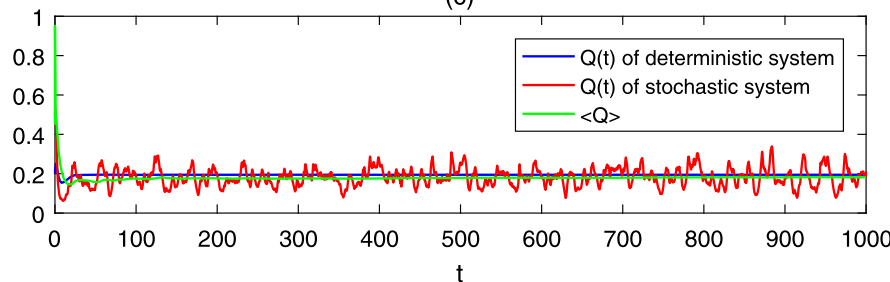

Figure 5 Comparison of system (2) and system (3), $u=0.32, \delta=0.58, \beta=0.3, b=0.2, q=0.26, \varepsilon=0.32$, $\alpha_{2}=0.12, \alpha_{3}=0.23, \gamma=0.25, m=0.01, n=0.5, A=0.72, \sigma=0.055$, where $R_{0}=1.3942>1, \widetilde{R}=1.3544>1$

(a)

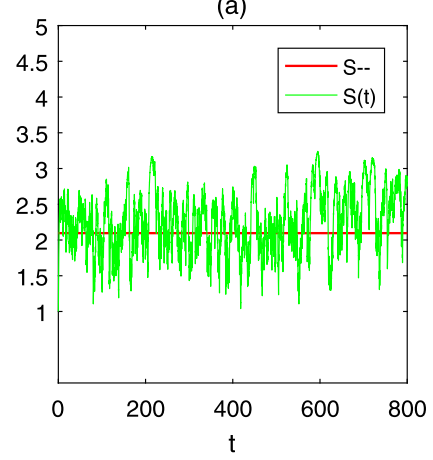

(c)

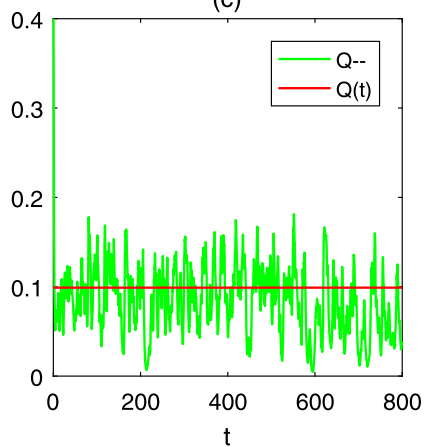

(b)

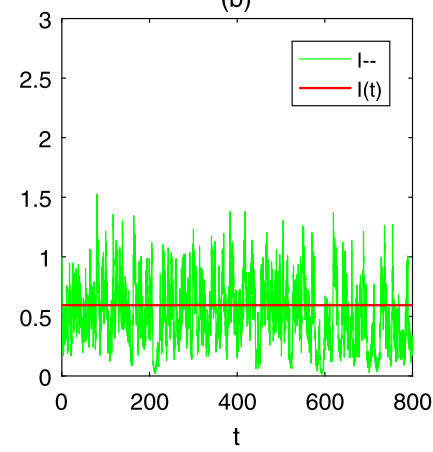

(d)

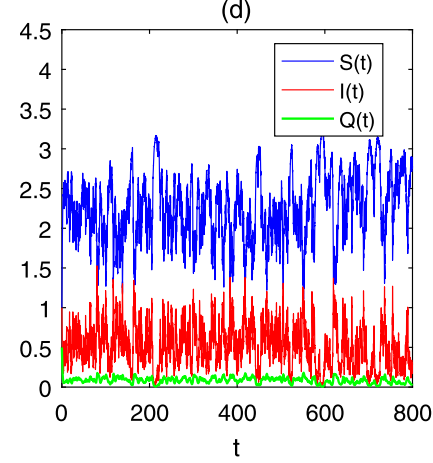

Figure 6 The oscillation behavior of system (3) near the endemic equilibrium point, $u=0.4, \delta=0.15, \beta=0.5$, $b=0.1, q=0.3, \varepsilon=0.35, \alpha_{2}=0.25, \alpha_{3}=0.15, \gamma=0.2, m=0.01, n=0.1, A=1, \sigma=0.2$, where $R_{0}=1.6628>1$, $\widetilde{R}=1.4482>1$ 


\section{Acknowledgements}

We thank the editor and the referees for their careful reading of the original manuscript and many valuable comments and suggestions that greatly improved the presentation of this paper.

\section{Funding}

This work is supported by Shandong Provincial Natural Science Foundation of China (No. ZR2019MA003), Research Funds for Joint Innovative Center for Safe and Effective Mining Technology and Equipment of Coal Resources by Shandong Province, and SDUST Research Fund (2014TDJH102).

\section{Availability of data and materials}

Data sharing not applicable to this article as all data sets are hypothetical during the current study.

\section{Competing interests}

The authors declare that they have no competing interests.

\section{Authors' contributions}

All authors worked together to produce the results and read and approved the final manuscript.

\section{Publisher's Note}

Springer Nature remains neutral with regard to jurisdictional claims in published maps and institutional affiliations.

\section{Received: 12 October 2019 Accepted: 2 July 2020 Published online: 14 July 2020}

\section{References}

1. World Health Organization: World health statistics 2016. Monitoring health for the SDGs Sustainable Development Goals. WHO, Geneva, Switzerland (2016)

2. Sehdev, P.S.: The origin of quarantine. Clin. Infect. Dis. 35(9), 1071-1072 (2002)

3. Wang, W., Ma, W., Feng, Z: Complex dynamics of a time periodic nonlocal and time-delayed model of reaction-diffusion equations for modeling CD4+ T cells decline. J. Comput. Appl. Math. 367, 112430 (2020)

4. Liu, Q., Jiang, D., Hayat, T., Alsaedi, A.: Dynamics of a stochastic multigroup SIQR epidemic model with standard incidence rates. J. Franklin Inst. 356(5), 2960-2993 (2019)

5. Wang, W., Cai, Y., Ding, Z., Gui, Z.: A stochastic differential equation SIS epidemic model incorporating Ornstein-Uhlenbeck process. Phys. A, Stat. Mech. Appl. 509, 921-936 (2018)

6. Cai, S., Cai, Y., Mao, X.: A stochastic differential equation SIS epidemic model with two independent Brownian motions. J. Math. Anal. Appl. 474(2), 1536-1550 (2019)

7. Zhang, X., Wang, X., Huo, H.: Extinction and stationary distribution of a stochastic SIRS epidemic model with standard incidence rate and partial immunity. Phys. A, Stat. Mech. Appl. 531, 121548 (2019)

8. Qi, H., Leng, X., Meng, X., Zhang, T.: Periodic solution and ergodic stationary distribution of SEIS dynamical systems with active and latent patients. Qual. Theory Dyn. Syst. 18(2), 347-369 (2019)

9. Ji, C., Jiang, D.: The extinction and persistence of a stochastic SIR model. Adv. Differ. Equ. 2017(1), 1 (2017)

10. Zhao, W., Liu, J., Chi, M., Bian, F.: Dynamics analysis of stochastic epidemic models with standard incidence. Adv. Differ. Equ. 2019(1), 22 (2019)

11. Feng, T., Qiu, Z., Meng, X.: Analysis of a stochastic recovery-relapse epidemic model with periodic parameters and media coverage. J. Appl. Anal. Comput. 9(3), 1007-1021 (2019)

12. Zhang, T., Wang, J., Li, Y., Jiang, Z., Han, X.: Dynamics analysis of a delayed virus model with two different transmission methods and treatments. Adv. Differ. Equ. 2020(1), 1 (2020)

13. Liu, K., Zhang, T., Chen, L.: State-dependent pulse vaccination and therapeutic strategy in an Sl epidemic model with nonlinear incidence rate. Comput. Math. Methods Med. 2019, Article ID 3859815, 10 pages (2019)

14. Wang, W., Ma, W., Feng, Z.: Dynamics of reaction-diffusion equations for modeling CD4+ T cells decline with general infection mechanism and distinct dispersal rates. Nonlinear Anal., Real World Appl. 51, 102976 (2020)

15. Dean, K.R., Krauer, F., Walløe, L., Lingjærde, O.C., Bramanti, B., Stenseth, N.C., Schmid, B.V.: Human ectoparasites and the spread of plague in Europe during the Second Pandemic. Proc. Natl. Acad. Sci. USA 115(6), 1304-1309 (2018)

16. Brunker, K., Mollentze, N.: Rabies virus. Trends Microbiol. 26(10), 886-887 (2018)

17. Mack, R.: The great African cattle plague epidemic of the 1890's. Trop. Anim. Health Prod. 2(4), 210-219 (1970)

18. Hethcote, H., Ma, Z., Liao, S.: Effects of quarantine in six endemic models for infectious diseases. Math. Biosci. $180(1-2), 141-160(2002)$

19. Wei, F., Chen, F.: Stochastic permanence of an SIQS epidemic model with saturated incidence and independent random perturbations. Phys. A, Stat. Mech. Appl. 453, 99-107 (2016)

20. Chen, L., Hu, Z., Liao, F.: The stability of an SEIRS model with Beddington-DeAngelis incidence, vertical transmission and time delay. J. Anhui Normal Univ. (2016, in press)

21. Kiouach, D., Sabbar, Y.: Stability and threshold of a stochastic SIRS epidemic model with vertical transmission and transfer from infectious to susceptible individuals. Discrete Dyn. Nat. Soc. 2018, Article ID 7570296 (2018)

22. Zhao, S., Yuan, S., Wang, H.: Threshold behavior in a stochastic algal growth model with stoichiometric constraints and seasonal variation. J. Differ. Equ. 268(9), 5113-5139 (2020)

23. Liu, G., Qi, H., Chang, Z., Meng, X.: Asymptotic stability of a stochastic May mutualism system. Comput. Math. Appl. 79(3), 735-745 (2020)

24. Yu, X., Yuan, S.: Asymptotic properties of a stochastic chemostat model with two distributed delays and nonlinear perturbation. Discrete Contin. Dyn. Syst., Ser. B 25(7), 2373-2390 (2017)

25. Gao, N., Song, Y., Wang, X., Liu, J.: Dynamics of a stochastic SIS epidemic model with nonlinear incidence rates. Adv. Differ. Equ. 2019(1), 41 (2019) ISSN 1687-1847 
26. Song, Y., Miao, A., Zhang, T., Wang, X., Liu, J.: Extinction and persistence of a stochastic SIRS epidemic model with saturated incidence rate and transfer from infectious to susceptible. Adv. Differ. Equ. 2018(1), 293 (2018) ISSN 1687-1847

27. Zhang, X., Huo, H., Xiang, H., Shi, Q., Li, D.: The threshold of a stochastic SIQS epidemic model. Phys. A, Stat. Mech. Appl. 482, 362-374 (2017)

28. Chi, M..ZZhao, W: Dynamical analysis of two-microorganism and single nutrient stochastic chemostat model with Monod-Haldane response function. Complexity 2019, Article ID 8719067 (2019)

29. Chang, Z., Meng, X., Zhang, T.: A new way of investigating the asymptotic behaviour of a stochastic SIS system with multiplicative noise. Appl. Math. Lett. 87, 80-86 (2019)

30. Zhang, Y., Fan, K., Gao, S., Liu, Y., Chen, S.: Ergodic stationary distribution of a stochastic SIRS epidemic model incorporating media coverage and saturated incidence rate. Phys. A, Stat. Mech. Appl. 514, 671-685 (2019)

31. Guo, W., Cai, Y., Zhang, Q., Wang, W.: Stochastic persistence and stationary distribution in an SIS epidemic model with media coverage. Phys. A, Stat. Mech. Appl. 492, 2220-2236 (2018)

32. Zhang, H., Zhang, T.: The stationary distribution of a microorganism flocculation model with stochastic perturbation. Appl. Math. Lett. 103, 106217 (2020) ISSN 0893-9659

33. Qi, H., Zhang, S., Meng, X., Dong, H.: Periodic solution and ergodic stationary distribution of two stochastic SIQS epidemic systems. Phys. A, Stat. Mech. Appl. 508, 223-241 (2018)

34. Zhao, W., Li, J., Zhang, T., Meng, X., Zhang, T.: Persistence and ergodicity of plant disease model with Markov conversion and impulsive toxicant input. Commun. Nonlinear Sci. Numer. Simul. 48, 70-84 (2017)

35. Liu, Q., Jiang, D., Shi, N.: Threshold behavior in a stochastic SIQR epidemic model with standard incidence and regime switching. Appl. Math. Comput. 316, 310-325 (2018)

36. Li, D., Liu, S., Cui, J.: Threshold dynamics and ergodicity of an SIRS epidemic model with Markovian switching. J. Differ. Equ. 263(12), 8873-8915 (2017)

37. Yu, X., Yuan, S., Zhang, T:. Survival and ergodicity of a stochastic phytoplankton-zooplankton model with toxin-producing phytoplankton in an impulsive polluted environment. Appl. Math. Comput. 347, 249-264 (2019)

38. Leng, X., Feng, T., Meng, X.: Stochastic inequalities and applications to dynamics analysis of a novel SIVS epidemic model with jumps. J. Inequal. Appl. 2017(1), 138 (2017)

39. Zhou, Y., Yuan, S., Zhao, D.: Threshold behavior of a stochastic SIS model with Lévy jumps. Appl. Math. Comput. 275 $255-267(2016)$

40. Zhang, X., Shi, Q., Ma, S., Huo, H., Li, D.: Dynamic behavior of a stochastic SIQS epidemic model with Lévy jumps. Nonlinear Dyn. 93(3), 1481-1493 (2018)

41. Liu, Q., Jiang, D., Hayat, T., Ahmad, B.: Analysis of a delayed vaccinated SIR epidemic model with temporary immunity and Lévy jumps. Nonlinear Anal. Hybrid Syst. 27, 29-43 (2018)

42. Gikhman, I.I., Skorokhod, A.V.: Stochastic Differential Equations and Their Applications. Naukova Dumka, Kiev (1982)

43. Miao, A., Zhang, T., Zhang, J., Wang, C.: Dynamics of a stochastic SIR model with both horizontal and vertical transmission. J. Appl. Anal. Comput. 8(4), 1108-1121 (2018)

44. Diekmann, O., Heesterbeek, J.A.P., Metz, J.A.: On the definition and the computation of the basic reproduction ratio $R_{0}$ in models for infectious diseases in heterogeneous populations. J. Math. Biol. 28(4), 365-382 (1990)

45. Yang, X., Li, F., Cheng, Y.: Global stability analysis on the dynamics of an SIQ model with nonlinear incidence rate. In: Advances in Future Computer and Control Systems, pp. 561-565 (2012)

46. Zhao, Y., Jiang, D.: The threshold of a stochastic SIS epidemic model with vaccination. Appl. Math. Comput. 243, 718-727 (2014)

47. Bian, F., Zhao, W., Song, Y., Yue, R.: Dynamical analysis of a class of prey-predator model with Beddington-Deangelis functional response, stochastic perturbation, and impulsive toxicant input. Complexity 2017, Article ID 3742197 (2017)

48. Higham, D.J.: An algorithmic introduction to numerical simulation of stochastic differential equations. SIAM Rev. 43(3), 525-546 (2001)

\section{Submit your manuscript to a SpringerOpen ${ }^{\circ}$ journal and benefit from:}

- Convenient online submission

Rigorous peer review

- Open access: articles freely available online

- High visibility within the field

- Retaining the copyright to your article

Submit your next manuscript at $\boldsymbol{~ s p r i n g e r o p e n . c o m ~}$ 